\title{
Terapias emergentes en artritis reumatoide
}

\author{
Juan C Aguillón G 1,a, Andrea Cruzat C ${ }^{1, b}$, \\ Juan Contreras-Levicoy ${ }^{1, b}$, Andrés D otte $G^{1, b}$, \\ Bárbara Pesce $R^{1, c}, 0$ ctavio Aravena $M^{1, c}$, \\ Lorena Salazar $A^{1, d}$, D iego Catalán $M^{1, d}$, Paula Abello $C^{1, e}$, \\ Adam Aguirre $D^{1, f}$, Carolina Llanos $M^{1,2, g}$, \\ Miguel Cuchacovich $\mathrm{T}^{2}$.
}

\section{Emergent therapies for rheumatoid arthritis}

The use of biological agents such as etanercept, infliximab, adalimumab and anakinra has been recently approved for the treatment of rheumatoid arthritis. All are effective controlling signs and symptoms and inhibiting disease progression. To overcome the problems generated by their high costs and possible participation in reactivating latent infections, other therapeutic tools are being developed. Gene therapy using expression vectors carrying genes coding for specific proteins, may interfere in key points involved in the pathogenesis of the disease. Intra-articular administration of cDNA coding for soluble TNF receptors, IL-1, or IL-1Ra decreases signs of the disease in animal models. Vectors, expressing inhibitors of signal transduction pathways involving to NF- $\kappa \mathrm{B}$ and JAK-STAT-3, are effective in modulating joint inflammation in mice. The use of antigen-pulsed antigen presenting cells or dendritic cells (DC) bound to apoptosis-inducing molecules, specifically eliminates autoreactive $\mathrm{T}$ cells. Other novel approach attempts the development of $\mathrm{T}$ regulatoryinducing tolerogenic DC-based vaccines that inhibit autoreactive T cells, through the secretion of suppressing cytokines or by other mechanisms to be elucidated. Oral tolerance induction to autoantigens is also a successful experimental strategy under study. Current research aims to control peripheral tolerance in rheumatoid arthritis patients (Rev Méd Chile 2005; 133: 969-76).

(Key Words: Arthritis, rheumatoid; Biological therapy; Gene therapy)

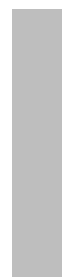

Recibido el 6 de octubre, 2004. Aceptado el 18 de enero, 2005.

Trabajo financiado por Proyectos: FONDEF DO311055 y FONDECYT 1040439 y 1040860.

${ }_{1}^{1}$ Programa Disciplinario de Inmunología, Instituto de Ciencias Biomédicas (ICBM), Facultad de Medicina, Universidad de Chile. ${ }^{2}$ Sección de Reumatología, Departamento de Medicina, Hospital Clínico de la Universidad de Chile, Santiago de Chile. ${ }^{\text {aPh.D. }}$ ${ }^{b}$ Estudiante de VII año de Medicina, Universidad de Chile. 'Licenciado en Bioquímica.

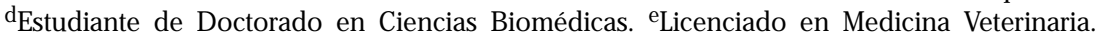

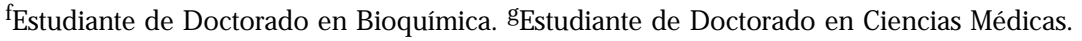

Correspondencia a: Juan C Aguillón, Ph.D. Programa Disciplinario de Inmunología, Instituto de Ciencias Biomédicas (ICBM), Facultad de Medicina, Universidad de Chile. Independencia 1027, Santiago, Chile. Tel: (56-2) 9786724. Fax: 562 735.3346. E mail: jaguillo@med.uchile.cl 
$\mathrm{D}$ urante los últimos años, se ha alcanzado un progreso substancial en el entendimiento de la inmunopatogénesis de diferentes enfermedades inflamatorias. Además, el progreso en biotecnología ha permitido el desarrollo de agentes biológicos que apuntan a moléculas y mecanismos específicos. Aun cuando es un hecho establecido, la participación de citoquinas proinflamatorias, tales como, interleuquina (IL)-1 e IL-6 en enfermedades reumatológicas y otros estados inflamatorios, el factor de necrosis tumoral (TNF) sería el principal efector regulador, teniendo una posición preponderante en la patogénesis de éstas. Por este motivo, en recientes estudios, se apunta a la neutralización específica de dichas citoquinas proinflamatorias, enfoque que ha tenido un éxito comprobado en enfermedades inflamatorias crónicas tales como artritis reumatoide ${ }^{1,2}$, enfermedad de $\mathrm{Crohn}^{3}$ y pelviespondilopatías ${ }^{4}$, entre otras.

\section{ARTRITIS REUMATOIDE}

La artritis reumatoide (AR) es una enfermedad sistémica autoinmune que afecta primariamente a la membrana sinovial y conduce a la destrucción de las articulaciones con degeneración del cartílago y erosión del hueso yuxta-articular como consecuencia de un proceso inflamatorio crónico.

Patogénesis y terapias biológicas. A partir de estudios en modelos animales ${ }^{5}$ y clínicos en pacientes con $\mathrm{AR}^{6}$, se descubrió que el TNF e IL-1 están directamente involucrados en la patogénesis de esta enfermedad. Gracias a un mejor entendimiento de los mecanismos fisiopatológicos que actúan en la AR, han aparecido nuevas armas terapéuticas que han modificado enormemente el enfrentamiento y abordaje de la enfermedad ${ }^{7}$. Estas nuevas terapias, conocidas como biológicas, antagonizan la acción del TNF e IL-1, las citoquinas iniciadoras de la cascada, que mantienen la inflamación sinovial y lideran la destrucción articular, cumpliendo un rol regulador sobre las otras citoquinas proinflamatorias, quimioquinas y factores de crecimiento expresados en las articulaciones afectadas. Han sido extensamente investigadas dos formas de inhibir el TNF como terapias en AR: anticuerpos monoclonales anti-TNF tanto quiméri$\cos$ (Infliximab) ${ }^{1,8}$ como completamente humanos
(Adalimumab) $^{2}$ y el receptor de TNF fusionado a un fragmento Fc de inmunoglobulina (Etanercept) ${ }^{9}$. Mientras que para IL-1 se ha desarrollado el antagonista de su receptor (IL-1Ra) de tipo recombinante (Anakinra) ${ }^{10}$.

Ensayos multicéntricos, randomizados, controlados con placebo, se han publicado recientemente, confirmando que el uso prolongado de agentes biológicos contra TNF como terapia en la AR es seguro, incrementa la mejoría en forma sostenida en los síntomas y signos de la enfermedad y la calidad de vida en $60-70 \%{ }^{11}$. Por lo anterior, los tres principales agentes anti-TNF: etanercept, infliximab y adalimumab, además de anakinra, han sido recientemente aprobados por la Food and Drugs Administration (FDA) de USA y la European Drug Authorities (EDA) en Europa ${ }^{12}$.

Mecanismo de acción. Al TNF se le atribuye un rol central en el inicio y perpetuación del daño crónico y la destrucción última, característica de la $\mathrm{AR}$, al controlar la cascada y promover la liberación de citoquinas proinflamatorias como IL-1, factor estimulante de colonias monocíticas-granulocíticas (GM-CSF) e IL-8. También induce la expresión de altos niveles de moléculas del complejo mayor de histocompatibilidad (MHC) de clase I, la síntesis y liberación de proteasas y prostaglandina E2 por fibroblastos sinoviales (lo que es importante en la erosión del cartílago y hueso), la neoangiogénesis sinovial $^{13}$ y eleva la cantidad de otros péptidos y mediadores lipídicos inflamatorios producidos en los órganos enfermos. El TNF también estimula varias otras células, incluyendo neutrófilos, fibroblastos sinoviales y condrocitos.

Los niveles de esta citoquina se encuentran elevados en el suero, el tejido y fluido sinovial de pacientes con $\mathrm{AR}^{14}$. Algunos trabajos sugieren que los monocitos de pacientes con AR temprana, sobreproducen TNF cuando están activados ${ }^{15}$. Ensayos clínicos en AR, han demostrado que el mecanismo de acción de los antagonistas de TNF incluyen una retroalimentación negativa de diversos agentes proinflamatorios, como IL-1, IL-6, IL-8, MCP-1 y la producción de VEGF, disminuye el reclutamiento de células inmunes e inflamatorias a las articulaciones, viéndose un efecto más general en los linfocitos $\mathrm{T}^{16}$, se regula la angiogénesis y se reducen los niveles séricos de metaloproteinasas de la matriz ${ }^{6}$. Se postula que la disminución sérica 
de moléculas de adhesión refleja la menor activación de células endoteliales en la microvasculatura sinovial, llevando a una reducción de la migración de los leucocitos a las articulaciones sinoviales y prolongando el efecto terapéutico de los bloqueadores de TNF en la $\mathrm{AR}^{17}$.

Otros estudios han demostrado claramente que bloqueando la actividad de TNF, se disminuye la reacción de fase aguda y hay menores niveles sistémicos y locales de moléculas de adhesión en pacientes con $\mathrm{AR}^{18}$.

La IL-1 constituye la segunda principal citoquina proinflamatoria en AR. IL-1Ra, su antagonista natural a nivel de su receptor, actúa de forma competitiva y permite bloquear la activación del receptor impidiendo la transducción de señales a nivel intracelular. Se ha demostrado que los pacientes con AR poseen niveles disminuidos de IL-1Ra en las articulaciones afectadas ${ }^{19}$.

Comparación con drogas modificadoras de la actividad reumática (DMARDS). La medición de la efectividad terapéutica de las nuevas drogas se realiza según los criterios definidos por la American College of Rheumatology (ACR) que incluyen: el número de articulaciones inflamadas y dolorosas, más una mejoría similar en al menos tres de los siguientes: dolor, evaluación general definida por el médico o el paciente, autoevaluación de inhabilidad física y niveles de reactantes de fase aguda. ACR20 se define como una reducción de $20 \%$ en los criterios mencionados, ACR50 como $50 \%$ o más, y ACR 70 como $70 \%$ o más ${ }^{20}$.

La terapia establecida actualmente con drogas antirreumáticas, como el metotrexato (MTX), sulfasalazina, hidroxicloroquina y leflunomida, que son aceptadas como la terapia sgold standard», llevan a cierta mejoría de los síntomas, pero son incapaces de detener la destrucción articular ${ }^{21}$. Son de acción lenta, ocasionalmente tóxicas y poco eficaces en casos severos. En estudios comparativos entre el tratamiento con etanercept y MTX en pacientes con $\mathrm{AR}$, el primero actúa más rápidamente en la disminución de síntomas y retarda el daño articular; además presenta menores efectos adversos e infecciones que $\mathrm{MTX}^{22}$. La terapia convencional para la $\mathrm{AR}$, sin ser antagonista específico del TNF, podría trabajar en parte, a través de la reducción, en algún grado, de los niveles de TNF. Así por ejemplo, los inhibidores de la ciclooxigenasa pueden atenuar muchos de los efectos agudos del $\mathrm{TNF}^{23}$ y los corticoesteroides, a través de su acción antiinflamatoria, en que inhiben parcialmente la producción de diversas citoquinas, incluyendo $\mathrm{TNF}^{24}$. Las distintas drogas anti-TNF y anti-IL-1, asociadas a MTX, tienen efecto beneficioso al ser comparadas contra placebo.

La combinación de etanercept y MTX fue significativamente mejor en reducir la actividad de la enfermedad, mejorar las limitaciones funcionales, y retardar la progresión radiológica de las lesiones óseas, al ser comparadas contra cada medicamento solo 25 .

En aquellos pacientes que mostraban respuesta incompleta al tratamiento con MTX solo, al agregar infliximab lograban una mejonía clínica significativa en su función física y calidad de vida, acompañado de inhibición de la progresión radiológica del daño articular, además, de una mejonía sustancial en los síntomas y signos de enfermedad (ACR20 de 48\%) al ser comparado con MTX solo (ACR20 de 16\%) ${ }^{26,27 .}$

Resultados similares se han observado en el uso de adalimumab ${ }^{28}$, el cual tiene efecto aditivo en las distintas dosis usadas, lográndose hasta $18 \%$ en ACR7029. En tanto, anakinra, al igual que los anti-TNF, al ser asociada con MTX es más eficaz que MTX solo, logrando hasta $6 \%$ de respuesta en ACR70 versus $2 \%$ con MTX solo ${ }^{10}$.

Por otra parte, todos los medicamentos antiTNF, al inhibir de algún modo la inmunidad celular, poseen el riesgo de tuberculosis u otra infección latente. Anakinra también ha mostrado conferir riesgo aumentado de infección, mayor si se combina con anti-TNF, además de producir neutropenia ${ }^{12}$. Pero claramente ésta constituiría una inmunodepresión mucho más selectiva en comparación a la masiva inhibición sobre el sistema inmunológico dada por las drogas de rutina, incrementándose de manera significativa el riesgo a infecciones oportunistas.

\section{NUEVAS ARMAS BIOLÓGICAS}

A pesar del beneficio aportado por los agentes biológicos antes mencionados, éstos poseen limitantes tales como: efectos secundarios sistémicos, reactivación de infecciones latentes, requerimiento de dosis repetidas para mantener su efecto y finalmente, lo exorbitante de su precio (aproximadamente US\$ 10.000/año/paciente). 
Lo antes enunciado, ha generado la necesidad del desarrollo de terapias alternativas basadas en enfoques diferentes e innovadores.

Terapia génica. Estudios en modelos animales han demostrado que la aplicación de la terapia génica es eficaz en el tratamiento de diversas patologías autoinmunes. Las potenciales ventajas de esta nueva terapia se basan en que los productos génicos pueden ser liberados localmente en el área afectada, mediante inyección intra-articular y transfección de células sinoviales. Lo anterior puede tener impacto en disminuir los efectos adversos, aumentar la selectividad, producto de transporte órgano-específico (mediante el uso de células-T, células presentadoras de antígeno o dendríticas, que luego de la inyección sistémica se localizan en el área de inflamación), y aumentar y mantener los niveles circulantes en el tiempo. Relevante a este último aspecto es el caso de la transfección de células musculares o hepáticas, cuyo producto se distribuye en forma sistémica.

Con el fin de dosificar la terapia, existen dos enfoques metodológicos: el primero, considera la extracción de las células blanco y luego de su manipulación en medios de cultivos, inyectarlas al sitio de interés (técnica ex vivo). En el segundo enfoque, se contempla la inyección directa de un vector, ya sea viral, plasmidial, o célula modificada genéticamente, que transporta ADN de interés, directamente al sitio en donde se requiere la expresión (técnica in vivo) ${ }^{30}$. Mediante el uso de esta tecnología, se puede expresar por varios meses en forma local (al transfectar sinoviocitos) o sistémica (al transfectar células musculares o hepáticas) distintos productos génicos que van a alterar diferentes puntos claves en la patogénesis de esta enfermedad.

De manera similar a lo que ocurre con la terapia antagonista de TNF, se ha observado que la administración intra-articular de cADNs codificantes de receptores solubles de TNF, de IL-1 e IL1Ra, disminuyen la intensidad de la enfermedad en modelos animales ${ }^{31}$.

Es un hecho demostrado que NF-кB y JAKSTAT-3, son las principales vías de transducción de señales intracelulares involucradas en la expresión de genes asociados con la inflamación y la destrucción tisular en AR. El uso de vectores con inhibidores de esta vía han mostrado eficacia en modelos animales, disminuyendo la severidad de la artritis y la inflamación articular (Figura 1) ${ }^{32,33}$.
Figura 1. Las vías de transducción NF$\kappa \mathrm{B}$ у MAPK son las principales activadas por citoquinas proinflamatorias tales como factor de necrosis tumoral (TNF) e interleuquina-1 (IL-1). La acción de moléculas antagonistas de TNF o IL-1 impiden la unión a sus receptores y como consecuencia la inducción de las cascadas de transducción de señales y subsiguiente activación de los factores de transcripción AP-1 y NF-кB. La actividad de las distintas quinasas y de los factores de transcripción constituye blancos terapéuticos que están siendo estudiados. AP-1, activator protein-1; IRAK, IL-1 receptor associated kinase; MAPK, mitogen-activated protein kinase; MyD88, myeloid differentiation protein 88; NEMO, NF- $\mathrm{kB}$ essential modulator; NF- $\mathrm{\kappa B}$, nuclear factor- $\kappa \mathrm{B}$; TRADD, TNF receptor-associated death domain protein; TRAF, TNF receptor-associated factor.

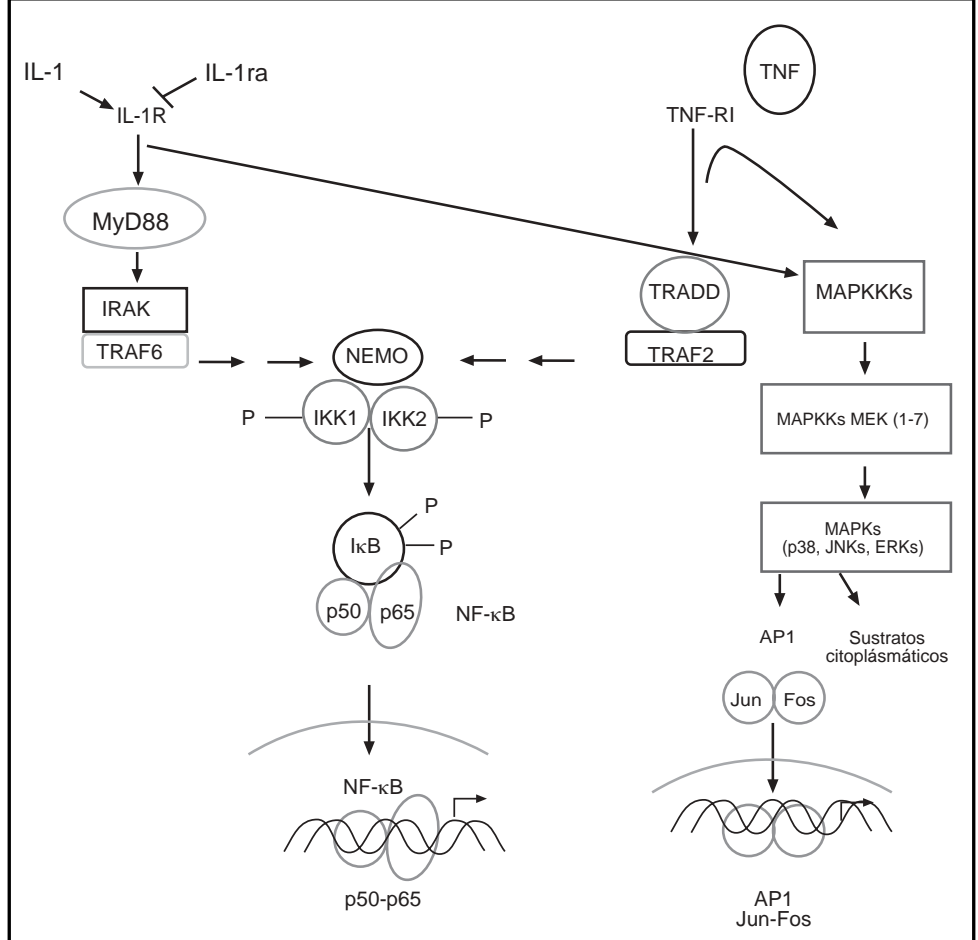


El pannus de las articulaciones contribuye a la destrucción articular, comportándose como una neoplasia. Se han diseñado estrategias para contrarrestarlo, destruyendo directamente células sinoviales, inhibiendo su división, induciendo apoptosis o interfiriendo con la circulación sanguínea de suministro. Mediante la inyección intraarticular de plasmidios de ADN codificantes de timidina quinasa del virus herpes simplex asociado a ganciclovir, se ha logrado en modelos animales una sinovectomía genética ${ }^{34}$. La inducción de apoptosis mediante el uso de p53 ha mostrado un efecto antiinflamatorio ${ }^{35}$. La inhibición de proliferación celular y los inhibidores angiogénicos también han tenido resultados satisfactorios en modelos animales.

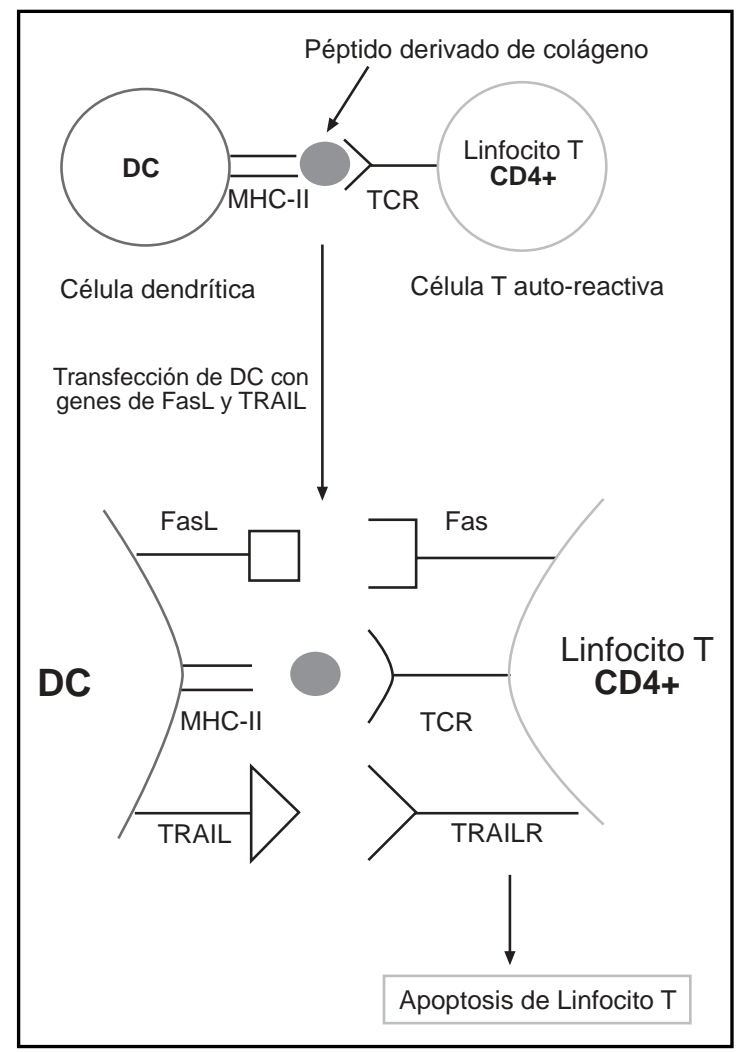

Figura 2. Inducción de apoptosis en células T $\mathrm{CD} 4^{+}$ auto-reactivas, mediada por la expresión de FasL y TRAIL en células dendríticas (DC). MHC II, major histocompatibility complex class II; TCR, antigen T cell receptor; TRAIL, TNF-related apoptosis-inducing ligand; TRAILR, TRAIL receptor protein.
Se ha descrito que a nivel de la articulación, las células $\mathrm{T}$ autorreactivas, tienen la propiedad de desplazarse hacia los sitios que poseen actividad inflamatoria, poseen una vida media prolongada y entran en proliferación una vez que se encuentran con el auto-antígeno específico. Por lo anterior, a estas células $\mathrm{T}$ se las puede considerar como buenos blancos de la terapia génica. Especialmente, por tratarse de una respuesta antígeno específica, no afectaría la respuesta inmunológica contra otras eventuales agresiones, como ha sido observado con la terapia anti-TNF.

Usando células T específicas de antígenos articulares y transfectadas con genes codificantes del receptor soluble de TNF, se ha logrado inhibir la enfermedad en las articulaciones ya afectadas, prevenir el avance de la enfermedad hacia nuevas articulaciones, y disminuir la actividad de las células $B$, efecto no logrado con las otras terapias anti-TNF ${ }^{30}$.

Terapia celular adoptiva: uso de células dendríticas. Dentro de la patogénesis de la $\mathrm{AR}$, se ha establecido la participación de células $\mathrm{T}$ autorreactivas contra una variedad de autoantígenos no bien definidos. Dichos antígenos son presentados a la célula $\mathrm{T}$ por una célula presentadora de antígeno, dentro de las cuales, las más importantes están constituidas por las células dendríticas, que interactúan en forma específica con la célula T autorreactiva (Figura 2).

Recientemente se ha logrado inducir la apoptosis de dichas células autorreactivas, mediante la interacción con un inductor de apoptosis. Dentro de éstos se encuentran Fas ligando (FasL) y el ligando inductor de apoptosis relacionado al TNF (tumor necrosis factor-related apoptosis-inducing ligand, TRAIL). Los receptores para ambas moléculas se encuentran en los linfocitos T. El vehículo utilizado para transportar estas señales es la célula dendrítica. En modelos murinos de artritis inducida por colágeno, se ha observado supresión de la enfermedad, al usar células dendríticas genéticamente modificadas para expresar FasL y TRAIL y pulsadas con colágeno, sin que se vean afectados los linfocitos $\mathrm{T}$ no autorreactivos, por tanto sin afectar el resto de la inmunidad (Figura 2).

Como se describe, las células dendríticas modificadas sólo inducen apoptosis en los linfocitos $\mathrm{T}$ que reconocen específicamente al colágeno desplegado en las moléculas MHC clase II presentes en la superficie de las células dendríticas. Este nuevo 
enfrentamiento, más específico, ha generado gran entusiasmo no solo en $\mathrm{AR}$, sino que en muchas enfermedades autoinmunes y cáncer. En el caso de AR se enfrenta la limitación que el antígeno contra el cual el sistema inmune se hace autorreactivo, aún es desconocido, y probablemente sean varios $^{36,37}$. En caso de identificarse tal autoantígeno es posible pensar en una cura para la AR.

Células dendríticas tolerogénicas. Las células dendríticas en el timo son responsables de la tolerancia central, mientras en los tejidos inducinán tolerancia periférica. Esta última, seńa mediada por células dendńticas inmaduras o semimaduras, que en ausencia de estímulo de maduración, presentańan antígenos tisulares a linfocitos $\mathrm{T}$, en un contexto subestimulatorio ${ }^{38}$. Uno de los mecanismos propuestos para ello, seńa la generación de células T reguladoras ( $\mathrm{Tr})$ que impiden la función de las células $\mathrm{T}$, a través de la secreción de citoquinas supresoras, además de otros mecanismos aún no dilucidados. Este mecanismo, ha sido recientemente aplicado al modelo de encefalitis experimental autoinmune, induciendo células Tr CD4+ productoras de IL-10 y demostrando su rol en la modulación de esta patología 39

Nuestro grupo de investigación, usando el modelo de artritis murina inducida por colágeno, se encuentra explorando el desarrollo de una terapia inmunorreguladora de la función de las células $\mathrm{T}$ autorreactivas. Así, se espera que la inoculación de células dendríticas murinas inmaduras o semimaduras, pulsadas con colágeno tipo II, induzca subpoblaciones de linfocitos T CD4+ antígenoespecíficos, productores de IL-10, que modifiquen tanto el curso de la artritis murina inducida por colágeno, como también el patrón de respuesta $\mathrm{T}$ «elper» 1, responsable de la perpetuación de la enfermedad y progresión del daño en la misma.

Inducción de tolerancia oral con péptidos antigénicos. Son varios los estudios orientados al tratamiento de AR, a través de la inducción de tolerancia oral, con diferentes auto-antígenos. Probablemente, el más utilizado ha sido el colágeno tipo II de distintos orígenes, obteniéndose respuestas clínicas de moderada magnitud ${ }^{40}$. Sin embargo, recientemente se ha informado de resultados bastante alentadores, mediante el uso de un péptido derivado de la proteína de «hock» térmico bacteriana, la cual presenta homología con cinco aminoácidos de la secuencia del «epitopo compartido», correspondiente a los residuos de la posición 70-74 de la cadena $ß$ de la molécula MHC II, que conforman la hendidura de unión peptídica de la molécula MHC y compartida esta especificidad alélica por un importante porcentaje de enfermos con AR. Los pacientes fueron tratados oralmente con este péptido, durante seis meses, observándose un quiebre de la respuesta $\mathrm{T}$ helper» 1 de las células T, expresado en un aumento de los niveles de producción de IL-4 e IL-10 y una disminución de los niveles de IFN $\gamma$, IL-2 y TNF, responsables estas últimas de la perpetuidad de la respuesta inflamatoria ${ }^{41}$.

\section{CONSIDERACIONES FINALES}

A pesar de lo reciente de la introducción al mercado de fármacos antagonistas de la actividad de citoquinas proinflamatorias, parece ser que éstos han mostrado ser relativamente seguros y efectivos, por lo menos en el corto y mediano plazo en el tratamiento de la AR. Por otra parte, ya son varias las aplicaciones de la terapia antagonista de TNF en el tratamiento de otras enfermedades, tales como espondilitis anquilosante, artritis juvenil, psoriasis, enfermedad de Still, uveitis y vasculitis ${ }^{7}$, mientras que otros usos se encuentran en investigación.

Cabe señalar, que durante las últimas dos décadas, el desarrollo de nuevas terapias para la $\mathrm{AR}$, ha sido un acontecimiento no frecuentemente observado en otras áreas. Hoy, la AR puede ser controlada adecuadamente en muchos pacientes de países desarrollados, siendo las manifestaciones extra-articulares como la vasculitis, situaciones más bien esporádicas. Esto ha sido posible con la incorporación de dosis más eficientes de metotrexato y sulfasalazina, o el uso de leflunomida, y especialmente, la introducción de los antagonistas de citoquinas, pero además, el consenso que existe en iniciar el tratamiento con drogas modificantes de la enfermedad reumática (DMARD), apenas se haya realizado el diagnóstico de la enfermedad.

Un hecho importante a destacar, es la disponibilidad de altemativas terapéuticas cuyo mecanismo de acción opera a distinto nivel, permitiendo probar más de una estrategia en un individuo y evitando llegar a las dosis máximas de las drogas habituales, ahorrándose la inmensa gama de efectos colaterales y adversos. Otro elemento interesante, es que varios de 
los enfoques hoy en estudio, apuntan a entender y solucionar el problema de la autoinmunidad, en términos mecanísticos, más que interferir en vías intermedias que no dan cuenta del origen del

\section{REFERENCIAS}

1. Euot MJ, Main RN, Feidmann M, Long-Fox A, Charies P, KatSIIIS P ET AL. Treatment of meumatoid arthritis with chimeric monoclonal antibodies to tumor necrosis factor $\alpha$. Arthritis Rheum 1993; 36: 1681-90.

2. Rankin EC, Choy EH, Kassimos D, Kingsley GH, SOPWITH AM, IsenBerg DA ET AL. The therapeutic effects of an engineered human anti-tumour necrosis factor alpha antibody (CDP571) in rheumatoid arthritis. Br J Rheumatol 1995; 34: 334-42.

3. Present DH, Rutgeerts P, Targan SR, Hanauer SB, MAYER L, VAN Hogezand RA ET AL. Infliximab for the treatment of fistulas in patients with Crohn's disease. N England J Med 1999; 340: 1398-405.

4. Braun J, De Keyser F, Brandt J, Mielants H, Sieper J, VEYs E. New treatment options in spondyloarthropathies: increasing evidence for significant efficacy of anti-tumor necrosis factor therapy. Curr Opin Rheumatol 2001; 13: 245-9.

5. Joosten LA, Helsen MM, Van De Loo fa, Van De BERG WB. Anticytokine treatment of established type II collagen-induced arthritis in DBA/1 mice. Arthritis Rheum 1996; 39: 797-809.

6. Feldmann M, Maini RN. The role of cytokines in the pathogenesis of rheumatoid arthritis. Rheumatology (Oxford) 1999; 38 Suppl 2: 3-7.

7. Aguilón JC, Contreras J, Dotte A, Cruzat a, Catalán D, Salazar L et al. New immunological weapons for medicine in the $21^{\text {st }}$ century: Biological therapy based on the use of the latest generation monoclonal antibodies. Rev Méd Chile 2003; 131: 1445-53.

8. Eunot MJ, Main RN, Feidmann M, Kaiden JR, Antoni C, SMolen JS et al. Randomized double-blind comparison of chimeric monoclonal antibody to tumour necrosis factor alpha (cA2) versus placebo in rheumatoid arthritis. Lancet 1994; 344: 1105-10.

9. Moreiand LW, SchifF MH, Baumgartner SW, TindalL EA, Fleischmann RM, BulpitT KJ et al. Etanercept therapy in rheumatoid arthritis. Ann Intern Med 1999; 130: 478-86.

10. Cohen SB, Moreland LW, Cush JJ, Greenwald MW, Block S, Shergy WJ et al. A multicentre, double blind, randomized, placebo controlled trial of anakinra (Kineret), a recombinant interleukin 1 problema. Tal es el caso de los estudios que se realizan a nivel del restablecimiento de la regulación de la función de los linfocitos T CD4+ automeactivos, basado en el uso de células dendníticas tolerogénicas.

receptor antagonist, in patients with rheumatoid arthritis treated with background methotrexate. Ann Rheum Dis 2004; 63: 1062-8.

11. KemPen J. Preliminary results of early clinical trials with the fully human anti-TNF $\alpha$ monoclonal antibody D2E7. Ann Rheum Dis 1999; 58 (suppl I): 170-2.

12. Olsen NJ, Stein CM. New drugs for rheumatoid arthritis. N Engl J Med 2004; 350: 2167-79.

13. Lupia E, Montrucchio G, Battaglia E, Modena V, Camussi G. Role of tumor necrosis factor- $\alpha$ and platelet-activating factor in neoangiogenesis induced by sinovial fluids of patients with rheumatoid arthritis. Eur J Immunol 1996; 26: 1690-4.

14. BRENNAN FM, Main RN, Feldmann M. TNF $\alpha$-pivotal role in rheumatoid arthritis? Br J Rheumatol 1992; 31: 293-8.

15. Leirisalo-Repo M, Paimeia L, JaAtTeia M, Koskimies $S$, REPO H. Production of TNF by monocytes of patients with early rheumatoid arthritis is increased. Scand J Rheumatol 1995; 24: 366-71.

16. RazA A. Anti-TNF therapies in rheumatoid arthritis, Crohn's disease, sepsis, and myielodisplastic syndromes. Microsc Res Tech 2000; 50: 229-35.

17. Tak PP, Taylor PC, Breedveld FC, Smeets TJ, Daha MR, KLUIN PM ET AL. Decrease in cellularity and expression of adhesion molecules by anti-tumor necrosis factor aaa monoclonal antibody treatment in patients with rheumatoid arthritis. Arthritis Rheum 1996; 39: 1077-81.

18. Brennan FM, Chantry D, Jackson A, Maini R, Feldmann M. Inhibitory effect of TNF alpha antibodies on synovial cell interleukin-1 production in rheumatoid arthritis. Lancet 1989; 2: 244-7.

19. Arend WP, Malyak M, Guthridge CJ, Gabay C. Interleukin-1 receptor antagonist: role in biology. Annu Rev Immunol 1998; 16: 27-55.

20. Feison DT, Anderson JJ, Botrs M, Bombardier C, Furst D, GoldSMTth C ET AL. American College of Rheumatology. Preliminary definition of improvement in meumatoid arthritis. Arthritis Rheum 1995; 38: 727-35.

21. Haraoui B, Strand V, Keystone E. Biologic agents in the treatment of rheumatoid arthritis. Curr Pharm Biotechnol 2000; 1: 217-33.

22. Bathon JM, Martin RW, Fieischmann RM, Tesser JR, Schiff MH, Keystone EC. A comparison of etaner- 
cept and methotrexate in patients with early rheumatoid arthritis. N England J Med 2000; 343: 1586-93.

23. Kettelhut IC, Fiers W, Goldberg AL The toxic effects of tumor necrosis factor in vivo and their prevention by ciclooxigenase inhibitors. Proc Natl Acad Sci USA 1987; 84: 4273-7.

24. Barrera P, Boerbooms AMT, Van De Putte, Van Der MEER JWM. Effects of antirheumatic agents on cytokines. Semin Arthritis Rheum 1996; 25: 234-53.

25. Kuareskog L, Van Der Heijde D, De Jager JP, Gough A, Kalden J, Malaise M et al. TEMPO (Trial of Etanercept and Methotrexate with Radiographic Patient Outcomes) study investigators. Therapeutic effect of the combination of etanercept and methotrexate compared with each treatment alone in patients with meumatoid arthritis: double-blind randomized controlled trial. Lancet 2004; 363: 675-81.

26. Main RN, Breedveld FC, Kalden JR, Smolen JS, Furst D, WeISMAN MH ET AL. Anti-tumor necrosis factor trial in rheumatoid arthritis with concomitant therapy study group. Sustained improvement over two years in physical function, structural damage, and signs and symptoms among patients with rheumatoid arthritis treated with infliximab and methotrexate. Arthritis Rheum 2004; 50: 1051-65.

27. Cuchacovich M, Ferreira L, Aliste M, Soto L, Cuenca J, CRuzAT A et al. Tumour necrosis factor-a (TNF-a) levels and influence of -308 TNF- $\alpha$ promoter polymorphism on the responsiveness to infliximab in patients with rheumatoid arthritis. Scand J Rheumatol 2004; 33: 228-32.

28. Keystone EC, Kavanaugh AF, Sharp JT, Tannenbaum H, Hua Y, Teон LS eт AL. Radiographic, clinical and functional outcomes of treatment with adalimumab (a human anti-tumor necrosis factor monoclonal antibody) in patients with active rheumatoid arthritis receiving concomitant methotrexate therapy: a randomized, placebo-controlled, 52-week trial. Arthritis Rheum 2004; 50: 1400-11.

29. Van De Putte LB, Atkins C, Malaise M, Sany J, Russell AS, Van Riel PL et al. Efficacy and safety of adalimumab as monotherapy in patients with rheumatoid arthritis for whom previous disease modifying antirheumatic drug treatment has failed. Ann Rheum Dis 2004; 63: 508-16.

30. RobBins PD, Evans CH, CHERNAJovskY Y. Gene therapy for arthritis. Gene Therapy 2003; 10: 902-11.
31. Gould DJ, Bright C, Chernajovsky Y. Inhibition of established collagen-induced arthritis with a tumour necrosis factor-alpha inhibitor expressed from a self-contained doxycycline regulated plasmid. Arthritis Res Ther 2004; 6: R103-13.

32. Zhang HG, Huang N, Lu D, Bibao L, Zhang X, Yang $P$ ET AL Gene therapy that inhibits nuclear translocation of nuclear factor kappaB results in tumor necrosis factor alpha-induced apoptosis of human synovial fibroblasts. Arthritis Rheum 2000; 43: 1094-105.

33. Shouda T, Yoshida T, Hanada T, Wakioka T, Oishi M, MiYoshi K ET AL. Induction of the cytokine signal regulator SOCS3/CIS3 as a therapeutic strategy for treating inflammatory arthritis. J Clin Invest 2001; 108: 1781-8.

34. Sant SM, Suárez TM, Moam MR, Wu BY, Blaivas M, LAING TJ ET AL. Molecular lysis of synovial lining cells by in vivo herpes simplex virus-thymidine kinase gene transfer. Hum Gene Ther 1998; 9: 2735-43.

35. Lu X, Fairbairn DW, Bradshaw WS, O'NewL KL, Ewert DL, SIMmons DL NSAID-induced apoptosis in Rous sarcoma virus-transformed chicken embryo fibroblasts is dependent on v-src and c-myc and is inhibited by bcl-2. Prostaglandins 1997; 54: 549-68.

36. Evans $\mathrm{CH}$. On the TRAIL of an arthritis cure. Gene Therapy 2004; 11: 735-6.

37. Kim SH, Kim S, Ougino TJ, Robbins PD. Effective treatment of established mouse collagen-induced arthritis by systemic administration of dendritic cells genetically modified to express FasL. Mol Ther 2002; 6: 584-90.

38. Steinman RM, Hawiger D, Nussenzweig MC. Tolerogenic dendritic cells. Annu Rev Immunol 2003; 21: 685-711.

39. Menges M, Rossner S, Voigtiander C, Schindier H, KuKUTSCh NA, Bogdan C ET aL. Repetitive injections of dendritic cells matured with tumor necrosis factor alpha induce antigen-specific protection of mice from autoimmunity. J Exp Med 2002; 195: 15-21.

40. Toussirot EA. Oral tolerance in the treatment of rheumatoid arthritis. Curr Drug Targets Inflamm Allergy 2002; 1: 45-2.

41. Prakken BJ, Samodal R, Le TD, Giannoni F, Yung GP, ScavUW J ET AL. Epitope-specific immunotherapy induces immune deviation of proinflammatory $\mathrm{T}$ cells in rheumatoid arthritis. Proc Natl Acad Sci USA 2004; 101: 4228-33. 